\section{Trabalhando com estratégias \\ de ensino-aprendizado por descoberta \\ na área da saúde: a problematização \\ e a aprendizagem baseada em problemas}

\author{
Discovery-based teaching and learning \\ strategies in health: problematization \\ and problem-based learning
}

Eliana Goldfarb Cyrino 1

Maria Lúcia Toralles-Pereira ${ }^{2}$

\footnotetext{
${ }^{1}$ Departamento de Saúde Pública, Faculdade de Medicina de Botucatu, Universidade Estadual Paulista, Botucatu, Brasil. 2 Departamento de Educação, Instituto de Biociências de Botucatu, Universidade Estadual Paulista, Botucatu, Brasil.

Correspondência Eliana Goldfarb Cyrino C. P. 549, Botucatu, SP 18618-970, Brasil. ecyrino@fmb.unesp.br
}

\begin{abstract}
Considering the changes in teaching in the health field and the demand for new ways of dealing with knowledge in higher learning, the article discusses two innovative methodological approaches: problem-based learning (PBL) and problematization. Describing the two methods' theoretical roots, the article attempts to identify their main foundations. As distinct proposals, both contribute to a review of the teaching and learning process: problematization, focused on knowledge construction in the context of the formation of a critical awareness; $P B L$, focused on cognitive aspects in the construction of concepts and appropriation of basic mechanisms in science. Both problematization and PBL lead to breaks with the traditional way of teaching and learning, stimulating participatory management by actors in the experience and reorganization of the relationship between theory and practice. The critique of each proposal's possibilities and limits using the analysis of their theoretical and methodological foundations leads us to conclude that pedagogical experiences based on PBL and/or problematization can represent an innovative trend in the context of health education, fostering breaks and more sweeping changes.
\end{abstract}

Health Education; Problem Based Learning; Higher Educational

\begin{abstract}
"Por que limitar-se a transmitir conhecimentos se os estudantes dispõem para isto, além da imprensa escrita, inventada há mais de 500 anos, outros meios de acesso às informações? Por que não privilegiar discussões em torno de temáticas levantadas junto aos alunos? Por que não prestigiar a aquisição de mentes criativas e inquiridoras, através de debates, de resoluções de problemas extraídos da própria realidade sóciocultural?" 1 (p. 178).

Há um reconhecimento internacional da necessidade de mudança na educação de profissionais de saúde frente à inadequação do aparelho formador em responder às demandas sociais. As instituições têm sido estimuladas a transformarem-se na direção de um ensino que, dentre outros atributos, valorize a eqüidade e a qualidade da assistência e a eficiência e relevância do trabalho em saúde. O processo de mudança da educação traz inúmeros desafios, entre os quais romper com estruturas cristalizadas e modelos de ensino tradicional e formar profissionais de saúde com competências que lhes permitam recuperar a dimensão essencial do cuidado: a relação entre humanos.

Almeida 2, em estudo recente sobre as possibilidades de mudança da Educação Médica, discute, com base em estudo de Ferreira 3, propostas de mudança relativas a alterações em processos, relações e conteúdos que, sinteticamente, podem ocorrer no plano da inovação, da reforma e da transformação.
\end{abstract}


Cunha et al. 4 , estudando os processos de mudanças em diferentes áreas, assinalam experiências inovadoras no limite de uma disciplina ou entre disciplinas de um mesmo curso, que podem contribuir para a melhoria do ensino e da aprendizagem nas universidades.

Na perspectiva desenvolvida por Cunha et al. 4 (p. 44), a inovação pode contribuir para a "ruptura com o paradigma dominante, fazendo avançar em diferentes âmbitos, formas alternativas de trabalhos que quebrem com a estrutura tradicional". Os autores observam, contudo, que uma inovação não se caracteriza simplesmente pelo uso de novos elementos tecnológicos no ensino, "a menos que estes representem novas formas de pensar o ensinar e o aprender, numa perspectiva emancipatória" 4 (p. 44). Uma experiência inovadora é um processo situado em um contexto histórico e social, que exige uma ruptura com procedimentos acadêmicos inspirados nos princípios positivistas da ciência moderna.

O movimento inovador no microinstitucional pode ser tão importante quanto o movimento do todo institucional, pois pode ocorrer "uma síntese (dialética) entre as condições objetivas institucionais e o surgimento dos interesses de mudança individuais" 5 (p. 72), favorecendo processos mais amplos. Assim, na contramão do modelo dominante de ensino e de experiências novas, desenvolvidas dentro de uma visão tecnicista e alienante, as ações inovadoras, que procuram explorar novas possibilidades no contexto dos conflitos e das contradições de uma escola historicamente situada, podem mobilizar processos significativos de mudança.

Considerando os processos de mudança da educação de profissionais de saúde e a demanda por novas formas de trabalhar com o conhecimento, busca-se, neste texto, compreender o trabalho do professor no âmbito da inovação pedagógica e as possibilidades de ruptura com base em dois caminhos, inerentes ao processo de ensino-aprendizagem, que têm ocupado o espaço de discussão sobre inovação na área da Saúde.

\section{Estratégias de ensino-aprendizagem problematizadoras: algumas reflexões}

Em 1998, diante da insatisfação com o currículo tradicional, no planejamento do curso de Saúde Coletiva da Faculdade de Medicina de
Botucatu, buscou-se uma possibilidade de ruptura por meio de duas metodologias problematizadoras. A partir dessa experiência e com base em trabalhos de pesquisadores, desenvolvemos uma reflexão sobre as possibilidades e limites de inovação pedagógica no ensino médico, mediante intervenções de âmbito metodológico.

Dentro das metodologias problematizadoras, a problematização e a aprendizagem baseada em problemas (ABP) são duas propostas distintas que "trabalham intencionalmente com problemas para o desenvolvimento dos processos de ensinar e aprender" 6 (p. 141). Apoiadas na aprendizagem por descoberta e significativa, ambas valorizam o aprender a aprender.

Venturelli 7, discutindo o processo educacional no mundo contemporâneo, resgata a necessidade de romper com a postura de transmissão de informações, na qual os alunos assumem o papel de indivíduos passivos, preocupados apenas em recuperar tais informações quando solicitados. Apropriando-se de conceitos desenvolvidos por Paulo Freire, ressalta a necessidade de conceber a educação como prática de liberdade, em oposição a uma educação como prática de dominação.

Ao propor a educação de adultos como prática de liberdade, Paulo Freire 8 defende que a educação não pode ser uma prática de depósito de conteúdos apoiada numa concepção de homens como seres vazios, mas de problematização dos homens em suas relações com o mundo. Por isso, a educação problematizadora fundamenta-se na relação dialógica entre educador e educando, que possibilita a ambos aprenderem juntos, por meio de um processo emancipatório.

A educação problematizadora trabalha a construção de conhecimentos a partir da vivência de experiências significativas. Apoiada nos processos de aprendizagem por descoberta, em oposição aos de recepção (em que os conteúdos são oferecidos ao aluno em sua forma final), os conteúdos de ensino não são oferecidos aos alunos em sua forma acabada, mas na forma de problemas, cujas relações devem ser descobertas e construídas pelo aluno, que precisa reorganizar o material, adaptando-o à sua estrutura cognitiva prévia, para descobrir relações, leis ou conceitos que precisará assimilar 9.

Ausubel (1978, apud Madruga 9) mostra que tanto a aprendizagem por recepção como por descoberta pode desenvolver-se de modo significativo ou repetitivo (mecânico). Para ser sig- 
nificativo, o conteúdo deve relacionar-se a conhecimentos prévios do aluno, exigindo deste uma atitude favorável capaz de atribuir significado próprio aos conteúdos que assimila, e do professor, uma tarefa mobilizadora para que tal aprendizagem ocorra 10 . Por outro lado, é repetitiva quando o aluno não consegue estabelecer relações do conteúdo novo com anteriores porque carece dos conhecimentos necessários para que tais conteúdos se tornem significativos ou não está mobilizado para uma aprendizagem ativa.

Na aprendizagem significativa, o aluno interage com a cultura sistematizada de forma ativa, como principal ator do processo de construção do conhecimento 11 . O ensino de novos conteúdos deve permitir que o aluno se desafie a avançar nos seus conhecimentos. Para isso, é necessário um trabalho de continuidade e ruptura em relação aos conhecimentos que o aluno traz. O conteúdo novo deve apoiar-se numa estrutura cognitiva já existente, o que exige do professor, como tarefa inicial, verificar o que o aluno sabe, para, de um lado, relacionar os novos conteúdos à experiência do aluno - a continuidade - e de outro, provocar novas necessidades e desafios pela análise crítica, levando o aluno a ultrapassar a sua experiência, os estereótipos, as sínteses anteriores etc. - é a ruptura 12.

$\mathrm{O}$ aprendizado é um processo complexo; não acontece de forma linear, por acréscimo, de modo a somar alguns novos elementos ao que sabíamos antes. Estrutura-se mediante redes de conexão que cada sujeito faz, "reelaborando associações singulares que se ampliame ganham novos sentidos à medida que é capaz de desenvolver novas relações, envolver-se na resolução de problemas que esclarecem novas questões abrindo-se para aprendizagens mais complexas" 13 (p. 46).

\section{Sobre a aprendizagem baseada em problemas}

A ABP tem como base de inspiração "os princípios da Escola Ativa, do Método Científico, de um Ensino Integrado e Integrador dos conteúdos, dos ciclos de estudo e das diferentes áreas envolvidas, em que os alunos aprendem a aprender e se preparam para resolver problemas relativos a sua futura profissão" 6 (p. 152). Penaforte 14 complementa essa definição lembrando que a busca das origens filosóficas da ABP encontra suas raízes na teoria do conhecimento do filósofo americano John Dewey, que se afirma entre o último decênio do século XIX e o terceiro decênio do século XX por meio de mo- vimentos como a Escola Nova e o movimento ativista.

Para Libâneo 12, o movimento da escola nova, inserido numa tendência pedagógica liberal progressista, surge por "razões de recomposição da hegemonia da burguesia" 12 (p. 20), como resposta ao ensino tradicional que valorizava o ensino humanista, no qual a interação professor-aluno não visava a qualquer relação com o cotidiano do estudante ou com a realidade social. Contrapondo-se ao modelo tradicional de ensino, o movimento da escola nova não chega a representar uma ruptura com o modelo de educação, em termos da relação política entre educação e sociedade; inova, no entanto, em termos de método, na forma de trabalhar com o conhecimento.

Na proposta educativa de Dewey, a aprendizagem parte de problemas ou situações que intencionam gerar dúvidas, desequilíbrios ou perturbações intelectuais. O método "dos problemas" valoriza experiências concretas e problematizadoras, com forte motivação prática e estímulo cognitivo para solicitar escolhas e soluções criativas 15.

Essa visão construtivista do conhecimento, apoiada na teoria piagetiana da equilibração e desequilibração cognitiva 16 , ganhou visibilidade no espaço escolar pelos estudos de Ferreiro 17 no âmbito da alfabetização. Contrapondo-se à prática corrente do ensino tradicional, a prática construtivista situa o professor no papel de provocar o raciocínio do aluno, procurando gerar desequilíbrios cognitivos (conflitos, problemas) em relação ao objeto de conhecimento a fim de possibilitar interações ativas com o conhecimento que levem o aluno a uma aprendizagem significativa. Neste caso, o aluno utiliza diferentes processos mentais (capacidade de levantar hipóteses, comparar, analisar, interpretar, avaliar), desenvolvendo capacidade de assumir responsabilidade por sua formação 11 .

A partir do pioneirismo da Universidade de McMaster, Canadá, no final dos anos de 1960, o modelo da ABP se expandiu para muitas escolas de Medicina em todo o mundo. Nota-se que, embora os pioneiros de McMaster não façam citação explícita a Dewey, é possível detectar na ABP fundamentos conceituais deste autor 14 . Muitas das características da APB já estavam presentes em uma reforma curricular da Escola de Medicina da Universidade de Case Western Reserve, nos anos de 1950, que incorporou e integrou um conjunto de métodos e estratégias instrucionais em laboratório multidisciplinar 18.

A Escola de Medicina de Harvard iniciou, em 1984, uma proposta curricular em ABP, im- 
plantada como programa curricular paralelo ao currículo tradicional, voluntário para estudantes de Medicina e para professores 19. Passados os primeiros anos e após uma avaliação comparativa entre o processo educacional tradicional e o novo currículo, Harvard passou a desenvolver um currículo único que "tinha como estratégias a $A B P$, o processo ensino-aprendizagem centrado no estudante e o resgate da relação médico-paciente como elemento agregador de conteúdos biopsicossociais" 20 (p. 164).

Ainda que outras escolas americanas, canadenses e européias já tivessem adotado inovações até mais radicais, a proposição de Harvard "marcou a incorporação de um novo modelo no cerne das instituições de ensino e pesquisa de indiscutível prestígio, gerando uma significativa pressão para mudanças em outras instituições" 20 (p. 164).

A ABP é, assim, considerada uma das mais significativas inovações na educação médica nos últimos anos, surgindo como um movimento de reação aos currículos das escolas médicas sob a forte influência do modelo flexineriano que privilegiava o modelo biomédico e o ensino centrado no hospital. Ela pode ser considerada como o eixo principal do aprendizado teórico de um currículo médico, objetivando o aprendizado de conteúdos cognitivos e a integração de disciplinas. Propõe-se a um trabalho criativo do professor que estará preocupado não só com $o$ "que", mas, essencialmente, com o "por que" e o "como" o estudante aprende.

\section{Construção de um currículo centrado na aprendizagem baseada em problemas}

A ABP é uma proposta de reestruturação curricular que objetiva a integração de disciplinas tendo em vista a prática. Para isso, organiza-se um elenco de situações que o aluno deverá saber/dominar, considerando o tipo de organização curricular. "Este elenco é analisado situação por situação para que se determine que conhecimentos o aluno deverá possuir para cada uma delas. São os denominados temas de estu$d o " 6$ (p. 145). Cada um destes temas de estudo será transformado em um problema para ser discutido em um grupo tutorial.

Assim, um problema é apresentado a um grupo de alunos por um professor tutor. Este problema, discutido em grupo, deve incentivar o levantamento de hipóteses para explicá-lo. A partir daí, objetivos serão traçados para melhor estudá-lo; pesquisas e estudos serão propostos e nova discussão em grupo será feita para síntese e aplicação do novo conhecimento. O tra- balho pode ocorrer de forma individual (cada aluno), mas também incentiva o trabalho em grupo como produto das atividades individuais. O grupo de tutoria constitui um fórum de discussão, apresentando-se como um laboratório que possibilita uma aprendizagem sobre a interação humana, constituindo-se numa oportunidade para aprender a ouvir, a receber e assimilar críticas 21 .

Os problemas são formulados e selecionados para serem apresentados a cada período do curso, no qual se espera que os estudantes consigam produzir alguma teoria, mas sempre com a proposta de que uma teoria mais completa seja buscada.

No Brasil, a Faculdade de Medicina de Marília (FAMEMA) e a Faculdade de Medicina do Centro de Ciências da Saúde da Universidade de Londrina (UEL) optaram por um currículo em ABP. Na FAMEMA, o programa de ensino/ aprendizagem centrado no estudante, baseado em problemas e orientado à comunidade, buscou romper a dicotomia entre os ciclos básico e clínico ${ }^{21}$. Na UEL, a mudança não se referiu ao método de ensino-aprendizado, mas a uma mudança de filosofia educacional que incluiu a integração de disciplinas de forma vertical e horizontal e a avaliação do estudante, não só somativa mas também formativa 22 .

A Escola de Saúde Pública do Ceará, no início dos anos 1990, iniciou um trabalho com a ABP na perspectiva de formar profissionais curiosos e impregnados pela idéia de compreender a necessidade do aprendizado contínuo 23 .

Nas últimas décadas, alguns autores passaram a se preocupar em entender as características de um currículo elaborado com base na $\mathrm{ABP}$, seus resultados e as ainda insolúveis questões para avaliar o impacto da ABP como uma nova proposta para a educação médica $24,25,26$. Porém, a literatura sobre a APB tem se caracterizado mais pela descrição de experiências do que por uma análise crítica sobre o paradigma da solução de problemas e os modelos curriculares que a utilizam 18.

\section{Trabalhando com a problematização}

A problematização tem, nos estudos de Paulo Freire 8 , a sua origem, enfatizando que os problemas a serem estudados precisam valer-se de um cenário real. Os problemas obtidos pela observação da realidade manifestam-se para alunos e professores com todas as suas contradições, daí o caráter fortemente político do trabalho pedagógico na problematização, marcado por uma postura crítica de educação. Edu- 
cação e investigação temática aparecem como momentos de um mesmo processo: o conteúdo deve estar sempre se renovando e ampliando, inserido criticamente na realidade; não uma realidade estática, mas em transformação, com todas as suas contradições. Criam-se, assim, desafios cognitivos permanentes para estudantes e professores.

Para Paulo Freire 8, quanto mais o professor possibilitar aos estudantes perceberem-se como seres inseridos no mundo, tanto mais se sentirão desafiados a responder aos novos desafios. Ensinar, aprender e pesquisar lidam com "dois momentos do ciclo gnosiológico: o em que se ensina e se aprende o conhecimento já existente e o em que se trabalha a produção do conhecimento ainda não existente" 27 (p. 31).

A metodologia da problematização é, também, uma das manifestações do construtivismo na educação. Mas está fortemente marcada pela dimensão política da educação, comprometida com uma visão crítica da relação educação e sociedade. Volta-se à transformação social, à conscientização de direitos e deveres do cidadão, mediante uma educação libertadora, emancipatória. Enquanto método, a problematização segue alguns passos de Dewey, entretanto, apresenta profundas resistências filosóficas e ideológicas. Dirige-se para a transformação das relações sociais pela prática conscientizadora e crítica.

Na problematização, a relação ação-reflexão-ação transformadora é o eixo básico de orientação do processo. Apoiada nos fundamentos da pedagogia crítica, a problematização tem por meta "o desenvolvimento da consciência crítica (...), e responde à essência de ser da consciência, que é a sua intencionalidade" 28 (p. 24).

Schall 29 cita a educadora Hortênsia de Hollanda, na década de 1950, como pioneira no campo da educação em Saúde, comparado-a aos trabalhos de Paulo Freire. Hortênsia de Hollanda buscou um novo conceito de educação em Saúde, incluindo a possibilidade de troca e de construção conjunta do conhecimento entre profissionais e população.

Diversos trabalhos, na área da Educação em Saúde, vêm utilizando-se da problematização. Mello et al. 30 realizaram estudo sobre o desenvolvimento de práticas de promoção à Saúde e Educação, em Quixadá, no Ceará, com a utilização da metodologia da pesquisa participante, articulando a construção do conhecimento ao agir para a resolução de problemas de interesse coletivo. Internacionalmente, na área da Saúde, metodologias problematizadoras surgiram na década de 1980 , em virtude da necessidade de buscar currículos orientados para problemas que melhor definissem como os estudantes aprendem e que habilidades cognitivas e afetivas estão sendo adquiridas. Uma proposta foi implementada na Universidade do Havaí, na Enfermagem, nomeada como “ensino baseado na investigação" (inquiry based learning), que inclui uma abordagem interdisciplinar de aprendizagem e solução de problemas, pensamento crítico e responsabilidade do aluno pela sua própria aprendizagem 18 .

No Brasil, diversas escolas de Enfermagem vêm trabalhando com a problematização. $\mathrm{Na}$ UEL, nos anos de 1990, iniciou-se um projeto especial de ensino, na área da Saúde, apoiado nessa metodologia. Há cerca de duas décadas, a problematização vem sendo usada na formação de auxiliares de enfermagem 6 .

Uma referência para utilização desta metodologia no Brasil, no ensino universitário, está presente nos trabalhos de Maguerez, Bordenave e Pereira, já na década de 1980. Os autores propõem um esquema de problematização da realidade, desenvolvido por Maguerez como método do arco, apoiado em cinco etapas: observação da realidade (problema) $\rightarrow$ pontoschave $\rightarrow$ teorização $\rightarrow$ hipóteses de solução $\rightarrow$ aplicação à realidade 31 .

O processo de ensino começa com a exposição dos alunos a um problema real: observação da realidade. Segue-se a "identificação das variáveis ou pontos-chave do problema, aqueles que, se modificados, poderiam resultar na solução do problema porque são os mais centrais..." 31 (p. 42). Na seqüência, os alunos buscarão uma teorização sobre o problema por intermédio de entrevistas com especialistas e pesquisas, buscando a contribuição da ciência para esclarecimento do estudo. Haverá, então, aplicação das hipóteses identificadas para a solução do problema, ocorrendo uma interação entre estudante e objeto de estudo, com intuito de um diálogo transformador para ambos 31 .

Para Berbel 6 (p. 144), a problematização "constitui uma verdadeira metodologia, entendida como um conjunto de métodos, técnicas, procedimentos ou atividades intencionalmente selecionados e organizados em cada etapa, de acordo com a natureza do problema em estudo e as condições gerais dos participantes".

Na educação problematizadora, busca-se interpretar a realidade voltando-se à criação de espaços contra-hegemônicos e contestatórios que possibilitem crítica, algumas vezes radical, à realidade estudada. Constatando e conhecendo os problemas, tornamo-nos capazes de intervir na realidade 27. Esta é a força da problematização para Paulo Freire: a possibilidade 
de romper com uma leitura de dominação. Como aponta Berbel 6 (p. 145), "está presente, nesse processo, o exercício da práxis e a possibilidade de formação da consciência da práxis".

\section{Aprendizagem baseada em problemas e problematização: duas propostas que se distinguem}

As duas propostas são, em seus núcleos teóricos constituintes, formulações distintas. A problematização tem como objetivo fundamental "a mobilização do potencial social, político e ético dos alunos, que estudam cientificamente para agir politicamente, como cidadãos e profissionais em formação, como agentes sociais que participam da construção da história de seu tempo, mesmo que em pequena dimensão" 6 (p. 145). É, portanto, uma proposta metodológica que propõe-se desvendar a realidade para transformá-la. Sua maior contribuição é a mudança de mentalidade, exigindo de todos os agentes sociais envolvidos no processo educativo a reavaliação de seus papéis, re-significando, coletivamente, o processo de ensino-aprendizagem. Há uma explicitação da intencionalidade política no ato de educar. A problematização insere-se numa concepção crítica de educação. A ABP, inspirada na Escola Nova, propõe-se a preparar cognitivamente os alunos para resolver problemas relativos a temas específicos do ensino da profissão.

Enquanto metodologia de ensino, a problematização pode ser utilizada para o ensino de determinados temas de uma disciplina, pela especificidade do objeto de estudo. Nem sempre é apropriada para todos os conteúdos. O desenvolvimento de uma prática apoiada na problematização não requer grandes mudanças materiais para sua implementação. A ABP é uma proposta que passa a direcionar toda a organização curricular de um curso, havendo necessidade de maior movimento do corpo docente, administrativo e acadêmico da instituição para desenvolvê-la. Sua utilização demanda alterações estruturais e trabalho integrado dos diversos departamentos e disciplinas que compõem o currículo dos cursos.

Tanto a ABP como a problematização têm, em comum, uma pergunta focal (o que está acontecendo?) como padrão para gerar novas informações, mediante processos de análise e síntese. Mas o ponto de partida, os pressupostos e a estrutura de trabalho desenvolvida, inclusive no campo afetivo, é diferente. O problema é mais abrangente na problematização, formulando-se pela observação de uma realidade com todas as suas contradições, enquanto na ABP o problema é apresentado aos alunos pelo professor-tutor 18.

A problematização é mais propícia para encorajar os alunos, em cada etapa de sua experiência de aprendizagem, a refletirem sobre a situação global de estudo de uma realidade concreta, com seus conflitos e contradições. Trata-se do estudo da realidade dinâmica e complexa, como observa Berbel 6 . Ambas ajudam os alunos a reverem seu processo de aprendizagem; todavia, a problematização volta-se com o fito de questionar o quanto determinada experiência mudou a compreensão, a apreensão, as atitudes e o comportamento de cada membro do grupo (alunos e professores), visando à consciência crítica e não apenas à compreensão dos conceitos e mecanismos básicos da ciência - objeto da ABP. Supera, portanto, o domínio cognitivo do conhecimento 18.

$\mathrm{Na} A B P$, os estudantes começam o estudo assumindo que o paciente (do problema elaborado no papel) tem um problema e que sua tarefa será encontrar a causa do mesmo procurando enfrentá-lo por diferentes caminhos do estudo. A intenção do caso escrito na $\mathrm{ABP}$, assim como o papel do tutor, é promover uma verdadeira investigação por parte dos estudantes, evitando tratar o problema como um mero exercício de raciocínio imediato, desencorajando o mau uso de referências no qual o aluno mata charadas. A intenção é promover a integração dos conteúdos utilizando diferentes processos mentais e ultrapassando a memorização de diagnósticos baseados em sinais e sintomas.

Nas duas formulações, os estudantes levantam as hipóteses para explicar o problema em estudo, e na problematização inclui-se um segundo momento de formulação de hipóteses para a solução e encaminhamento do problema, como possibilidade de propor uma intervenção na realidade.

O problema do cenário na $\mathrm{ABP}$, por ser construído no papel visando articular conteúdos definidos, pode, às vezes, ficar descolado da realidade, enquanto a problematização, apresentada em um cenário real, amplia a possibilidade de percepção da própria realidade.

O ensino realizado com a metodologia da problematização pode ser entendido como possível de ser aplicada tanto em um planejamento curricular como no planejamento de um curso, de uma disciplina, ou, até mesmo, para o ensino de determinados temas de uma disciplina. Na ABP há de se rever conteúdos, possibilidades de integração dos conteúdos e/ou disciplinas e, ainda, abrir-se ao debate sobre o que é essencial para o aprendizado do estu- 
dante no currículo. Evidenciam-se, assim, as freqüentes dificuldades encontradas por professores quando trabalham com a ABP de forma isolada, em uma disciplina ou em um conjunto de disciplinas, no contexto de um currículo marcadamente tradicional.

A problematização requer do professor uma mudança de postura para o exercício de um trabalho reflexivo com o aluno, exigindo a disponibilidade do professor de pesquisar, de acompanhar e colaborar no aprendizado crítico do estudante, o que freqüentemente coloca o professor diante de situações imprevistas, novas e desconhecidas, exigindo que professores e alunos compartilhem de fato o processo de construção (e não apenas o de reconstrução e reelaboração) do conhecimento. Na ABP, o problema e os seus objetivos de aprendizado já estão definidos para o professor-tutor antecipadamente; seu trabalho refere-se mais a dar seqüência aos objetivos na discussão dos problemas. Nas duas proposições há uma mudança radical no papel do professor, que passa a não ser mais o centro do processo de ensino.

Na problematização, há a necessidade não só de uma clara postura metodológica em relação ao processo de pesquisa que o aluno deverá desenvolver ativamente, mas também de uma coerente postura política em relação ao processo educativo e aos problemas relativos ao tema em estudo. Pelas características do trabalho com o conhecimento, após o estudo de um problema poderão surgir outros desdobramentos sobre o tema, exigindo do professor e dos alunos o contato com situações ou conteúdos que não foram previstas pelo professor, num primeiro momento, mas que precisarão ser investigadas por serem relevantes à compreensão do problema. Os conhecimentos científicos, buscados no momento da teorização, integram percepções, conhecimentos, representações de pessoas envolvidas no problema, permitindo que diferentes saberes sejam conjugados no processo de construção do conhecimento. Na ABP, os objetivos cognitivos são todos previamente estabelecidos, devendo os objetivos levantados pelos alunos coincidir com os dos especialistas do currículo, caso contrário, os problemas devem ser substituídos 6 .

Berbel 6 observa que há uma diferença quanto ao uso dos resultados em cada uma das proposições. Na ABP, os novos conhecimentos são usados para aquisição de habilidades, exercício e construção intelectual. Na problematização, os resultados deverão voltar-se para a compreensão crítica da realidade estudada, tendo em vista a sua transformação mediante proposições de intervenção na realidade estudada.

\section{Movimentos de mudança no ensino}

Discutindo os rumos das Escolas Médicas no Brasil e as propostas de mudanças curriculares, Briani 32 ressalta que mudanças têm sido desenvolvidas numa perspectiva tecnicista, privilegiando a organização do currículo a uma orientação restrita à atividade técnica - o como fazer. Essas propostas voltam-se à promoção de metodologias de ensino centradas no aluno, na resolução de problemas; porém, tendem a desenvolver experiências copiadas, sem reflexão crítica, de modelos de ensino de outras nações, não conseguindo problematizar a organização do currículo no contexto da sociedade contemporânea, nem discutir demandas econômicas, políticas ou questões ideológicas envolvidas na formulação desses currículos.

Acreditamos que experiências pedagógicas, aparentemente pontuais, geradas no contexto dos conflitos e das contradições das relações institucionais, voltadas para a mudança de processos, relações e conteúdos, podem representar um movimento inovador em termos do processo de reelaboração de um conhecimento mais significativo para os alunos, favorecendo rupturas com o modelo tradicional de ensino capazes de levar a movimentos mais amplos de mudança. Tanto a problematização como a ABP promovem rupturas com a forma tradicional de ensinar e aprender, estimulando gestão participativa dos protagonistas da experiência e reorganização da relação teoria e prática, mas têm potenciais diferentes em termos de concepção de educação. Perceber criticamente as possibilidades e limitações de cada uma destas propostas, valendo-se da análise de seus fundamentos teóricos e metodológicos, considerando, ainda, as condições reais de cada instituição (características do curso e das diferentes disciplinas que o integram, metas e princípios pedagógicos que orientam o processo de formação, a organização curricular e o trabalho do professor em sala de aula com os alunos) representa uma possibilidade de buscar modelos de ensino-aprendizagem que respondam às expectativas da comunidade docente de uma determinada instituição em termos de possibilidades e de limitações que essa instituição apresenta, num determinado momento, para lidar com as demandas por mudança. 


\section{Resumo}

Considerando os processos de mudança no ensino em saúde e a demanda por novas formas de trabalhar com o conhecimento no ensino superior, discutem-se dois caminhos metodológicos inovadores no ensino na área da saúde: a aprendizagem baseada em problemas $(A B P)$ e a problematização. Descrevendo suas raízes teóricas, procura-se identificar os seus princípios orientadores. Enquanto propostas distintas, ambas contribuem para rever o processo de ensino-aprendizagem: a problematização, voltando-se para a construção do conhecimento no contexto de uma formação crítica; a $A B P$, voltando-se para os aspectos cognitivos do processo de construção de conceitos e apropriação dos mecanismos básicos da ciência. Tanto a problematização como a ABP levam a rupturas com a forma tradicional de ensinar e aprender, estimulando gestão participativa dos protagonistas da experiência e reorganização da relação teoria/prática. A crítica às possibilidades e limites de cada proposta, valendo-se da análise de seus fundamentos teórico-metodológicos, leva-nos a concluir que experiências pedagógicas apoiadas na ABP elou na problematização, podem representar um movimento inovador no contexto da educação na área da saúde favorecendo rupturas e processos mais amplos de mudança.

Educação em Saúde; Aprendizado Baseado em Problemas; Ensino Superior

\section{Colaboradores}

E. G. Cyrino e M. L. Toralles-Pereira desenvolveram em conjunto as idéias do artigo. Ambas as autoras foram responsáveis por todas as etapas de construção do artigo.

\section{Referências}

1. Balzan NC. Formação de professores para o ensino superior: desafios e experiências. In: Bicudo MA, organizador. Formação do educador e avaliação educacional. São Paulo: Editora UNESP; 1999. p. 173-88.

2. Almeida MJ. Educação médica e saúde: possibilidades de mudança. Londrina: EDUEL/Rio de Janeiro: Associação Brasileira de Educação Médica; 1999.

3. Ferreira JR. Análisis prospectivo de la educación médica. Educ Med Salud 1986; 20:26-42.

4. Cunha MI, Marsico HL, Borges FA, Tavares P. Inovações pedagógicas na formação inicial de professores. In: Fernandes CMB, Grillo M, organizadores. Educação superior: travessias e atravessamentos. Canoas: Editora da ULBRA; 2001. p. 33-90.

5. Leite D. Pedagogia universitária: conhecimento, ética e política no ensino superior. Porto Alegre: Editora da UFRGS; 1999.

6. Berbel NAN. A problematização e a aprendizagem baseada em problemas. Interface Comun Saúde Educ 1998; 2:139-54.

7. Venturelli J. Educación médica: nuevos enfoques, metas y métodos. Washington, DC: Organización Panamericana de la Salud/Organización Mundial de la Salud; 1997.

8. Freire P. Pedagogia do oprimido. Rio de Janeiro: Paz e Terra; 1975.

9. Madruga A. Aprendizagem pela descoberta frente à aprendizagem pela recepção: a teoria da aprendizagem verbal significativa. In: Coll C, Palácios J, Marchesi A, organizadores. Desenvolvimento psicológico e educação. Porto Alegre: Artes Médicas; 1996. p. 68-78.

10. Coll C. Um marco de referência psicológico para a educação escolar: a concepção construtivista da aprendizagem e do ensino. In: Coll C, Palácios J, Marchesi A, organizadores. Desenvolvimento psicológico e educação. Porto Alegre: Artes Médicas; 1996. p. 389-404.

11. Cunha MI. Ensino com pesquisa: a prática do professor universitário. Cadernos de Pesquisa 1996; 97:31-46.

12. Libâneo JC. Democratização da escola pública. A pedagogia crítico-social dos conteúdos. São Paulo: Loyola; 1987.

13. Ribeiro ECO. Ensino/aprendizagem na escola médica. In: Marcondes E, Gonçalves E, organizadores. Educação médica. São Paulo: Sarvier; 1998. p. 40-9.

14. Penaforte J. John Dewey e as raízes filosóficas da aprendizagem baseada em problemas. In: Mamede S, Penaforte J, Schmidt H, Caprara A, Tomaz JB, Sá H, organizadores. Aprendizagem baseada em problemas: anatomia de uma nova abordagem educacional. Fortaleza: Escola de Saúde Pública/São Paulo: Editora Hucitec; 2001. p. 49-78.

15. Cambi F. História da pedagogia. São Paulo: Editora UNESP; 1999.

16. Piaget J. A equilibração das estruturas cognitivas. Rio de Janeiro: Zahar; 1976.

17. Ferreiro E. Reflexões sobre alfabetização. 5a Ed. São Paulo: Cortez; 1986.

18. Feletti G. Inquiry based and problem based learn- 
ing; how similar are these approaches to nursing and medical education. Higher Education Research and Development 1993; 12:143-56.

19. Moore GT. Initiating problem-based learning at Harvard Medical School. In: Boud D, Felleti G, editors. The challenge of problem based learning. New York: St. Martin's Press; 1991. p. 80-7.

20. Aguiar AC. Implementando as novas diretrizes curriculares para educação médica: o que nos ensina o caso de Harvard? Interface Comun Saúde Educ 2001; 5:161-6.

21. Komatsu RS, Zanolli M, Lima VV. Aprendizagem baseada em problemas. In: Marcondes E, Gonçalves E, organizadores. Educação médica. São Paulo: Sarvier; 1998. p. 223-37.

22. Gordon P. A new medicine curriculum in Londrina: the Dean's perspective on the change process project. Chicago: Department of Medical Education, College of Medicine, University of Illinois at Chicago; 2001.

23. Mamede S, Penaforte J, Schmidt H, Caprara A, Tomaz JB, Sá H. Aprendizagem baseada em problemas: anatomia de uma nova abordagem educacional. Fortaleza: Escola de Saúde Pública/São Paulo: Editora Hucitec; 2001.

24. Bligh J. Editorials problem-based learning: the story continues to untold. Med Educ 2000; 3:688-9.

25. Colliver JA. Effectiveness of problem-based learning curricular: research and theory. Acad Med 2000; 75:259-66.
26. Albanese M. Problem based learning: why curricular are likely to show little effect on knowledge and clinical skills. Med Educ 2000; 34:729-38.

27. Freire P. Pedagogia da autonomia: saberes necessários à prática educativa São Paulo: Paz e Terra; 1996.

28. Berbel NAN, organizador. A metodologia da problematização e os ensinamentos de Paulo Freire: uma relação mais que perfeita. In: Metodologia da problematização: fundamentos e aplicações. Londrina: EDUEL; 1999. p. 1-28.

29. Schall V. Alfabetizando o corpo: o pioneirismo de Hortênsia de Hollanda na educação em saúde. Cad Saúde Pública 1999; 15 (Suppl 2):149-60.

30. Mello DA, Rouquayrol MZ, Araújo D, Amadei M, Souza J, Bento LF, et al. Promoção à saúde e educação: diagnóstico de saneamento através da pesquisa participante articulada à educação popular. Cad Saúde Pública 1998; 14:583-95.

31. Bordenave JD, Pereira AM, organizadores. O que é ensinar. In: Estratégias de ensino-aprendizagem. Petrópolis: Vozes; 2000. p. 39-57.

32. Briani MC. O ensino médico do Brasil está mudando? Rev Bras Educ Med 2001; 25:73-7.

Recebido em 31/Out/2002

Versão final reapresentada em 28/Out/2003

Aprovado em 11/Nov/2003 